\title{
Fruit quality of jabuticaba progenies cultivated in a tropical climate of altitude
}

Mayara Neves Santos Guedes ${ }^{1 *}$, José Carlos Moraes RufinI², Alcinei Mistico AzeVedo², Nísia Andrade Villela Dessimoni PINTo³

${ }^{1}$ UFSJ-Agric. Dep., Sete Lagoas Campus, Rodovia, MG 424, km 47, Sete Lagoas, MG,

Brazil,

maysantos2@yahoo.com.br

2 UFV-Plant Sci. Dep.,

Univ. Campus, Viçosa, MG, Brazil

3 UFVJM-Nutr. Dep., JK Campus, Rod MG 367, $\mathrm{km} 583, \mathrm{n}^{\circ} 5000$,

Alto da Jacuba, MG, Brazil

${ }^{*}$ Correspondence and reprints

Received 5 January 2014 Accepted 27 March 2014

Fruits, 2014, vol. 69 , p. 449-458 (C) 2014 Cirad/EDP Sciences All rights reserved

DOI: 10.1051/fruits/2014030 www.fruits-journal.org

RESUMEN ESPAÑOL, p. 458

\section{Fruit quality of jabuticaba progenies cultivated in a tropical climate of altitude.}

Abstract - Introduction. The jabuticaba tree (Myrciaria jabuticaba) belongs to the Myrtaceae family and produces a small fruit, native to central, southern and southeastern Brazil. Materials and methods. Fruits of 25 progenies of the jabuticaba tree, grown in a tropical highland climate, were evaluated as to physical, physicochemical and chemical characteristics: weight, longitudinal and transverse diameters, pulp and peel yields, seed mass, number of seeds per fruit, pulp and peel moisture, soluble solids, titratable acidity, [soluble solids/titratable acidity] ratio, $\mathrm{pH}$, flavonoids, and phenolic compounds. The experimental design was completely randomized, the treatments being the 25 jabuticaba tree progenies. Results. A significant effect among progenies was observed for all characteristics evaluated. The chemical composition showed that the jabuticaba peel has a high phenolic compound content, and considerable flavonoid content. These results indicate that there is variability for the characteristics analyzed, enabling the economic exploitation of jabuticaba tree fruits for in natura consumption and processing. Conclusions. The physical and chemical characteristics combined can be proposed as excellent tools to select superior progenies. The fruits of five progenies showed desirable characteristics for the in natura market, while fruits of five other progenies hold high concentrations of natural antioxidants and may be listed as excellent options for the food industry.

Brazil / Myrciaria jabuticaba / fruits / physicochemical properties / flavonoids / phenolic content / processing

\section{Qualité du fruit de descendances de jabuticabas cultivés dans un climat tropical d'altitude.}

Résumé - Introduction. Le jabuticaba (Myrciaria jabuticaba) appartient à la famille des myrtacées ; il produit de petits fruits et est indigène aux régions centre, sud et sud-est du Brésil. Matériel et méthodes. Des fruits de 25 descendances de jabuticaba, développées en climat tropical montagneux, ont été évalués quant à leurs caractéristiques physiques, physicochimiques et chimiques : poids, diamètres longitudinal et transversal du fruit, rendements en peau et pulpe, masse de graines, nombre de graines par fruit, taux d'humidité de la pulpe et de la peau, teneurs en solides solubles, acidité titrable, taux [solides solubles/acidité titrable], pH, teneurs en flavonoïdes et composés phénoliques. Le dispositif expérimental a été complètement randomisé, les traitements étant constitués par les 25 descendances de jabuticaba étudiées. Résultats. Un effet significatif de toutes les caractéristiques évaluées a été observé parmi les descendances. La composition chimique a révélé que la peau du fruit de jabuticaba a une teneur élevée en composés phénoliques, et un taux en flavonoïdes considérable. Nos résultats indiquent qu'il existe une variabilité parmi les caractéristiques analysées, justifiant une exploitation économique des fruits du jabuticaba pour sa consommation en frais et pour son industrialisation. Conclusion. L'étude combinée des caractéristiques physiques et chimiques peut être proposée comme un excellent outil pour sélectionner des descendances supérieures. Les fruits de cinq descendances ont montré des caractéristiques intéressantes pour le marché du fruit frais, alors que les fruits de cinq autres descendances présentant de fortes teneurs en antioxydants naturels pourraient être retenus comme une bonne option pour l'industrie alimentaire.

Brésil / Myrciaria jabuticaba / fruits / propriété physicochimique / flavonoïde / teneur en phénols / traitement 


\section{Introduction}

The jabuticaba tree belongs to the Myrtaceae family, one of the most important fruitbearing families occurring in Brazil. This fruit tree is found in a wide range throughout the country, from Pará to Rio Grande do Sul; however, the states of the Southeast have the highest production [1]. The jabuticaba tree is relatively tall, flowers emerging directly in small nodules on the trunk or branches [2]. It produces a berry type fruit of about $3-4 \mathrm{~cm}$, containing 1 to 4 seeds; the peel is black when ripe. The pulp, thin and fragile, is whitish with a sweet, slightly acid flavor [3]. The jabuticaba fruit pulp and peel present high concentrations of phenolic compounds with a significant antioxidant function [4]. To determine the quality of the fruit, various parameters can be adopted, those that are physical, such as mass, length, diameter and shape; or chemical such as soluble solids, $\mathrm{pH}$, titratable acidity, phenolics, flavonoids and others [5]. The fruit with standard weight and size characteristics are easier to handle in bulk, as they present less loss, higher yield and better quality [6].

To control the maturity level of the fruit, the monitoring of soluble solids, which indicates the amount of solids that are dissolved in the juice, and acidity, which determines the percentage of organic acids and is one of the main methods to measure acidity of fruit, has been used [7]. The $\mathrm{pH}$ is the most workable method to determine the quality of processed products [8].

Characteristics related to climate adaptation to diverse jabuticaba tree-growing regions and physical and chemical properties of its fruits are divergent; variations may notably occur in the physical and physicochemical quality and content of chemical compounds in function of the locale where they are grown; this is due to the differences in the solar radiation intensity and temperature range, which influence the organoleptic characteristics of fruit [6].

Studies evaluating the genetic diversity and selection of superior materials have intensified in recent years. Physical and chemical attributes evaluated in eight jabuticaba tree clones certified significant variation in fruit shape and, even in relation to the fruits of the 'Sabara' jabuticaba tree, it was not possible to state that its spherical shape is determinant [2]. Another study found that growing conditions, natural or in shade, of the jabuticaba tree did not affect fruit quality [9]. For the Carica papaya, genetic variability was due to the physical characteristics of fruits [10]. A study of apples showed that their quality was affected by different growth conditions, both in relation to primary and secondary metabolites [11].

Thus, our study aimed to perform biometric, physicochemical and chemical characterization of jabuticaba fruits from a tropical climate region of altitude, seeking the selection of promising jabuticaba tree progenies (Myrciaria jabuticaba) for the fresh fruit market and agro-processing.

\section{Materials and methods}

Our study was conducted at the Federal University of the Jequitinhonha and Mucuri Valleys (UFVJM), Diamantina, Minas Gerais. Diamantina belongs to the Mesoregion of the Jequitinhonha Valley and is located on the southern ridge region, with predominantly rocky and sandy soils, with genesis in quartzite rocks and those with low moisture- (water) retaining power. The climate is $\mathrm{Cwb}$, according to the Köppen classification, i.e., tropical of altitude with summer rains and cool summers according to Cupolillo [12].

Fruits of 25 jabuticaba tree progenies (Myrciaria jabuticaba) from DiamantinaMG, pre-selected as to plant vigor and health, were collected manually and randomly in various positions and orientations on the plants at the commercial maturity stage. The fruits were transported to the Postharvest Laboratory, where they were selected for the formation of experimental units.

The experimental design was completely randomized, with 25 treatments (jabuticaba tree progenies) and four replications. 


\subsection{Quantitative evaluation of morphological characteristics}

Longitudinal and transverse diameters were analyzed with a caliper; the [longitudinal/ transverse] diameter ratio, fruit mass, peel, pulp and seed percentages, and number of seeds per fruit were measured.

\subsection{Evaluation of physicochemical and chemical characteristics}

\subsubsection{Moisture}

Moisture in the pulp and peel was determined by drying in a forced-air oven at a temperature of $(65 \pm 5){ }^{\circ} \mathrm{C}$ for $48 \mathrm{~h}$ according to the AOAC [13].

\subsubsection{Soluble solids}

The soluble solids content in the pulp and peel of each progeny was evaluated with the aid of a refractometer (Warszawa PZO-RL $3^{\circledR}$, Poland) and the results were corrected for $20{ }^{\circ} \mathrm{C}$.

\subsubsection{Titratable acidity}

The titratable acidity determination was performed by titration with sodium hydroxide solution $0.1 \mathrm{~N}$ standardized using phenolphthalein as an indicator according to the Adolfo Lutz Institute-IAL [14] (g citric acid $100 \mathrm{~g}^{-1}$ fresh sample).

\subsection{4. [Soluble solids/titratable acidity] ratio}

The ratio of total soluble solids (TSS) to total titratable acidity (TTA), expressed in ${ }^{\circ}$ Brix/ $\mathrm{mg} \%$, was calculated by the [TSS/TTA] ratio.

\subsection{5. $\mathrm{pH}$}

The $\mathrm{pH}$ was determined by direct reading on a digital potentiometer according to the Adolfo Lutz Institute-IAL [14].

\subsubsection{Flavonoids}

The extraction of the flavonoids was carried out in $80 \%$ methanol and quantified by spectrophotometry according to Zhuang et al. [15]; the results were expressed as $\mathrm{mg}$ catechin $\cdot 100 \mathrm{~g}^{-1}$ of fresh fruit.

\subsubsection{Total phenols}

The extraction of phenolic compounds was performed according to Goldstein and Swain [16] and measured by the FolinDenis method according to the AOAC [13]; the results were expressed in $\mathrm{g}$ tannic acid $100 \mathrm{~g}^{-1}$ of fresh fruit.

\subsection{Statistical design}

The statistical design was completely randomized, the treatments containing 25 accessions of the jabuticaba tree 'Sabara', with ten fruits per plot for the physical characteristics and four repetitions for chemical characteristics. Data were analyzed statistically and means were compared using the Scott-Knott test, adopting 5\% probability. For statistical analysis we used the Sisvar program [17].

\section{Results and discussion}

\subsection{Morphological characteristics}

According to the statistical analysis, for all variables of morphological characteristics of fruits, there was the formation of different numbers of groups (table I), which indicates the capability of some variables of differentiating genetic differences among the 25 jabuticaba tree progenies.

The mass of the jabuticaba tree (Myrciaria jabuticaba) fruit grown in a tropical climate of altitude region averaged $3.86 \mathrm{~g}$ per fruit (table I), lower than the values found in different cultivation locations by Lima et al. [18], Citadin et al. [9] and Oliveira et al. [1], which were $9.16 \mathrm{~g}, 7.23 \mathrm{~g}$ and $5.47 \mathrm{~g}$, respectively. Values closer to those observed in our study were found by Jesus et al. [19], who observed fresh fruit mass from (1.60 to 4.50) $\mathrm{g}$ in jabuticaba trees under Jabuticabal-SP conditions, and fruits obtained by Hickel averaged $4.62 \mathrm{~g}$ for the jabuticaba tree (Myrciaria cauliflora) [20].

The longitudinal and transversal diameter values ranged from (15.23 to 22.56$) \mathrm{mm}$ and (14.22 to 22.07$) \mathrm{mm}$, respectively 
M.N.S. Guedes et al.

Table I.

Mean values of fruit mass, longitudinal and transverse diameters, longitudinal and transverse diameter ratio [LD/TD], pulp and peel percentages, seed percentage, number of seeds, and pulp and peel moisture of jabuticaba tree fruit (Brazil).

\begin{tabular}{|c|c|c|c|c|c|c|c|c|c|c|}
\hline \multirow[t]{2}{*}{ Progenies } & \multirow{2}{*}{$\begin{array}{l}\text { Fruit mass } \\
\text { (g) }\end{array}$} & \multicolumn{2}{|c|}{ Diameter } & \multirow{2}{*}{$\begin{array}{c}{[\mathrm{LD} / \mathrm{TD}]} \\
\text { ratio }\end{array}$} & \multirow{2}{*}{$\begin{array}{l}\text { Pulp } \\
\text { (\%) }\end{array}$} & \multirow{2}{*}{$\begin{array}{l}\text { Peel } \\
(\%)\end{array}$} & \multirow{2}{*}{$\begin{array}{c}\text { Seed } \\
(\%)\end{array}$} & \multirow{2}{*}{$\begin{array}{l}\text { Number } \\
\text { of seeds }\end{array}$} & \multicolumn{2}{|c|}{ Moisture } \\
\hline & & $\begin{array}{l}\text { Longitudinal } \\
\text { (LD) } \\
(\mathrm{mm})\end{array}$ & $\begin{array}{c}\text { Transverse } \\
\text { (TD) } \\
\text { (mm) }\end{array}$ & & & & & & $\begin{array}{l}\text { Pulp } \\
(\%)\end{array}$ & $\begin{array}{c}\text { Peel } \\
(\%)\end{array}$ \\
\hline$P_{1}$ & $2.16 \mathrm{~h}$ & 18.27 e & $18.23 d$ & $1.00 \mathrm{c}$ & $65.42 \mathrm{~b}$ & $20.68 \mathrm{e}$ & $13.88 \mathrm{a}$ & $1.21 \mathrm{C}$ & $84.68 \mathrm{c}$ & $79.03 \mathrm{~d}$ \\
\hline $\mathrm{P}_{2}$ & $5.11 \mathrm{c}$ & $18.26 \mathrm{e}$ & $18.19 \mathrm{~d}$ & $1.00 \mathrm{c}$ & $73.66 \mathrm{a}$ & $17.97 \mathrm{e}$ & $8.36 \mathrm{c}$ & $2.65 \mathrm{a}$ & $84.87 \mathrm{c}$ & $81.41 \mathrm{c}$ \\
\hline$P_{3}$ & $5.29 \mathrm{c}$ & 18.29 e & $18.85 \mathrm{c}$ & $0.97 d$ & $72.13 \mathrm{a}$ & $18.24 \mathrm{e}$ & $9.62 \mathrm{~b}$ & $2.82 \mathrm{a}$ & $87.53 \mathrm{a}$ & $85.95 \mathrm{a}$ \\
\hline $\mathrm{P}_{4}$ & $3.47 \mathrm{f}$ & $17.91 \mathrm{e}$ & $17.62 \mathrm{~d}$ & $1.01 \mathrm{~b}$ & $64.72 \mathrm{~b}$ & $26.95 d$ & $8.31 \mathrm{c}$ & $1.52 \mathrm{~b}$ & $84.49 c$ & $83.82 \mathrm{~b}$ \\
\hline $\mathrm{P}_{5}$ & $2.59 \mathrm{~h}$ & $16.62 \mathrm{f}$ & $16.33 \mathrm{e}$ & $1.02 \mathrm{~b}$ & $58.56 \mathrm{c}$ & $28.55 b$ & $12.88 \mathrm{a}$ & $2.85 \mathrm{a}$ & $84.87 \mathrm{c}$ & $80.40 \mathrm{c}$ \\
\hline$P_{6}$ & $2.32 \mathrm{~h}$ & $15.93 \mathrm{~g}$ & $15.59 \mathrm{e}$ & $1.02 \mathrm{~b}$ & $58.61 \mathrm{c}$ & $29.85 \mathrm{~b}$ & $11.54 b$ & $2.27 \mathrm{~b}$ & $83.79 \mathrm{~d}$ & $80.54 \mathrm{c}$ \\
\hline$P_{7}$ & $2.21 \mathrm{~h}$ & $15.38 \mathrm{~g}$ & $14.22 \mathrm{e}$ & $1.08 \mathrm{a}$ & $60.83 c$ & $28.42 \mathrm{~b}$ & $10.73 b$ & $1.65 b$ & $86.26 \mathrm{~b}$ & $83.47 \mathrm{~b}$ \\
\hline $\mathrm{P}_{8}$ & $2.24 \mathrm{~h}$ & $15.40 \mathrm{~g}$ & $15.23 \mathrm{e}$ & $1.01 \mathrm{~b}$ & $54.14 \mathrm{c}$ & $34.92 \mathrm{a}$ & $10.93 \mathrm{~b}$ & $1.77 \mathrm{~b}$ & $85.98 \mathrm{~b}$ & $81.34 \mathrm{c}$ \\
\hline$P_{9}$ & $5.28 \mathrm{c}$ & $20.69 \mathrm{c}$ & $20.62 \mathrm{~b}$ & $1.00 \mathrm{c}$ & $60.42 \mathrm{c}$ & $31.53 \mathrm{~b}$ & $8.03 \mathrm{c}$ & $1.75 b$ & $84.29 d$ & $81.33 \mathrm{c}$ \\
\hline$P_{10}$ & $5.97 \mathrm{~b}$ & $21.57 \mathrm{~b}$ & $21.49 a$ & $1.00 \mathrm{c}$ & $64.75 \mathrm{~b}$ & $27.42 \mathrm{~d}$ & $7.82 \mathrm{c}$ & $1.12 \mathrm{c}$ & $87.65 \mathrm{a}$ & $83.82 \mathrm{~b}$ \\
\hline$P_{11}$ & $6.51 \mathrm{a}$ & $22.56 \mathrm{a}$ & $22.07 \mathrm{a}$ & $1.02 \mathrm{~b}$ & $62.67 \mathrm{c}$ & $29.80 \mathrm{~b}$ & $7.51 \mathrm{c}$ & $1.10 \mathrm{c}$ & $83.98 d$ & $80.24 \mathrm{c}$ \\
\hline$P_{12}$ & $4.17 \mathrm{e}$ & $18.82 \mathrm{~d}$ & $18.83 \mathrm{c}$ & $0.99 \mathrm{c}$ & $60.10 \mathrm{c}$ & $33.66 \mathrm{a}$ & $6.23 d$ & $1.47 \mathrm{~b}$ & $87.65 \mathrm{a}$ & $83.57 \mathrm{~b}$ \\
\hline$P_{13}$ & $3.56 f$ & $17.97 \mathrm{e}$ & $17.77 \mathrm{~d}$ & $1.01 \mathrm{~b}$ & $57.21 \mathrm{c}$ & $35.62 \mathrm{a}$ & $7.16 \mathrm{c}$ & $1.52 b$ & $85.21 \mathrm{c}$ & $81.40 \mathrm{c}$ \\
\hline$P_{14}$ & $2.30 \mathrm{~h}$ & $15.58 \mathrm{~g}$ & $15.27 \mathrm{e}$ & $1.02 \mathrm{~b}$ & $58.08 \mathrm{c}$ & $30.16 \mathrm{~b}$ & $7.75 b$ & $1.72 b$ & $85.30 \mathrm{c}$ & $81.23 \mathrm{c}$ \\
\hline$P_{15}$ & $4.77 \mathrm{~d}$ & $18.96 \mathrm{~d}$ & $18.89 \mathrm{c}$ & $1.00 \mathrm{c}$ & $62.93 c$ & $29.12 \mathrm{~b}$ & $7.93 \mathrm{c}$ & $1.07 \mathrm{c}$ & $85.97 \mathrm{~b}$ & $82.62 \mathrm{~b}$ \\
\hline$P_{16}$ & $2.99 \mathrm{~g}$ & $15.23 \mathrm{~g}$ & $14.91 \mathrm{e}$ & $1.02 \mathrm{~b}$ & $55.36 \mathrm{c}$ & $35.48 \mathrm{a}$ & $9.15 \mathrm{c}$ & $1.77 \mathrm{~b}$ & $83.70 \mathrm{~d}$ & $79.09 d$ \\
\hline$P_{17}$ & $4.07 \mathrm{e}$ & $20.21 \mathrm{c}$ & $20.33 b$ & $0.99 \mathrm{c}$ & $60.69 \mathrm{c}$ & $33.28 \mathrm{a}$ & $6.02 \mathrm{~d}$ & $1.40 \mathrm{c}$ & $85.56 \mathrm{~b}$ & $80.97 \mathrm{c}$ \\
\hline$P_{18}$ & $4.86 \mathrm{~d}$ & $18.73 d$ & $18.71 \mathrm{c}$ & $1.00 \mathrm{c}$ & $60.25 c$ & $34.45 \mathrm{a}$ & $5.30 \mathrm{~d}$ & $1.35 \mathrm{c}$ & $83.07 \mathrm{e}$ & $80.49 c$ \\
\hline$P_{19}$ & $4.82 \mathrm{~d}$ & $17.81 \mathrm{e}$ & $17.62 \mathrm{~d}$ & $1.01 \mathrm{~b}$ & $69.63 \mathrm{a}$ & $26.49 d$ & $3.87 \mathrm{e}$ & $1.22 \mathrm{c}$ & $81.11 \mathrm{f}$ & $75.95 \mathrm{f}$ \\
\hline$P_{20}$ & $4.77 \mathrm{~d}$ & $19.62 \mathrm{~d}$ & $20.10 \mathrm{~b}$ & $0.97 d$ & $60.82 \mathrm{c}$ & $30.60 \mathrm{~b}$ & $6.56 \mathrm{~d}$ & $1.55 b$ & $84.78 \mathrm{c}$ & $81.15 \mathrm{c}$ \\
\hline$P_{21}$ & $3.80 \mathrm{f}$ & $19.48 d$ & $19.76 d$ & $0.98 d$ & $69.28 \mathrm{c}$ & $30.01 \mathrm{~b}$ & $7.70 \mathrm{c}$ & $1.75 b$ & $85.10 \mathrm{c}$ & $82.33 \mathrm{~b}$ \\
\hline$P_{22}$ & $4.60 \mathrm{~d}$ & $19.44 d$ & $19.90 \mathrm{~b}$ & $0.97 d$ & $67.37 \mathrm{~b}$ & $25.57 d$ & $7.06 \mathrm{c}$ & $1.62 b$ & $83.37 \mathrm{e}$ & $77.34 \mathrm{e}$ \\
\hline$P_{23}$ & $2.98 \mathrm{~g}$ & 17.89 e & $18.23 d$ & $0.98 d$ & $63.43 c$ & $26.04 d$ & $10.52 b$ & $1.90 \mathrm{~b}$ & $82.62 \mathrm{e}$ & $79.53 d$ \\
\hline $\mathrm{P}_{24}$ & $2.11 \mathrm{~h}$ & $19.02 \mathrm{~d}$ & $18.95 \mathrm{c}$ & $1.00 \mathrm{c}$ & $60.20 \mathrm{c}$ & $25.57 \mathrm{~b}$ & $11.08 \mathrm{~b}$ & $1.32 \mathrm{c}$ & $84.88 \mathrm{c}$ & $81.27 \mathrm{c}$ \\
\hline$P_{25}$ & $3.70 \mathrm{f}$ & $16.62 \mathrm{f}$ & $16.63 \mathrm{e}$ & $0.99 c$ & $67.36 \mathrm{~b}$ & $25.57 d$ & $7.05 \mathrm{c}$ & $1.35 \mathrm{c}$ & $84.13 d$ & $80.48 c$ \\
\hline $\begin{array}{l}\text { Coefficient } \\
\text { of variation }\end{array}$ & 9.25 & 3.17 & 3.24 & 1.31 & 7.25 & 13.06 & 14.46 & 15.06 & 0.771 & 1.00 \\
\hline Mean & 3.86 & 18.25 & 18.18 & 1.00 & 62.54 & 28.76 & 8.68 & 1.67 & 84.83 & 81.15 \\
\hline
\end{tabular}

In the columns, means followed by the same letter belong to a common group, according to the Scott-Knott criterion of grouping $(P<0.05)$.

(table I). These values are consistent with those found by Oliveira et al., which range from (17.70 to 24.70$) \mathrm{mm}$ in longitudinal diameter and (17.30 to 24.50$) \mathrm{mm}$ in transverse diameter of the jabuticaba tree (Myrciaria jabuticaba) fruits [1]. Citadin et al. [9] and Hickel [20] observed, respectively, diameters of $24.22 \mathrm{~mm}$ and $19.97 \mathrm{~mm}$ for jabuticaba tree fruit (Myrciaria cauliflora). Fruits of the progenies P11 and P10 showed the highest mass and diameters (table I).

The pulp yield ranged from $54.14 \%$ to $73.66 \%$ (table I). Twelve percent of the progenies showed fruit with pulp yield above $69 \%$. The yield of pulp is an important quality characteristic for the industry [6]. 
The values found in our study are in accordance with the results found by Soprano and Koller, who obtained $53.70 \%$ of pulp yield for jabuticaba [21]; however, they differ from the values reported by Lima et al. [18] and Sato and Cunha [22], who observed on average $36.00 \%$ and $21.70 \%$ of fruit pulp, respectively.

The jabuticaba tree accessions that presented the highest percentage of fruit peel had values between $33.28 \%$ and $35.62 \%$ (table I). The behavior of the $\mathrm{P}_{2}$ and $\mathrm{P}_{3}$ progenies regarding the peel percentage was contrary to the pulp percentage. The jabuticaba tree fruits are preferred for in natura consumption when they have thin peel, and thus a larger amount of pulp. Similar results for the peel fraction were found by Oliveira et al. for 'Sabara' jabuticaba fruit from different growing regions, with an average of $32.40 \%$ [1]. Lima et al. observed that the peel fraction represented $43.00 \%$ of the fruits of the 'Sabara' jabuticaba tree [18], higher than the value found in our work. The jabuticaba peel consists primarily of crude protein and dietary fiber, and can be used as an alternative source of food or as an ingredient for obtaining processed preparations [23].

The average seed content percentage of 'Sabara' jabuticaba tree progenies was $8.68 \%$. Thirty-two percent of the progenies evaluated presented between $13.88 \%$ and $9.62 \%$ (table I). In our work, the peel and seed fraction together represent approximately $37.44 \%$ of the 'Sabara' jabuticaba tree fruits, while Lima et al. found $18.00 \%$ and $50.00 \%$, respectively, for the peel fraction, and peel and seed fraction together [18].

The number of seeds per fruit ranged from 1.07 to 2.85 , with $40 \%$ of the progenies varying from 1.07 to 1.40 (table I). Soprano and Koller observed 1.96 to 3.82 seeds per jabuticaba tree fruit from the states of Piauí and Maranhão [21]. The percentage and number of seeds is related to the size of the fruit and, consequently, yield and quality.

The moisture results indicated that the pulp and peel, after dehydration, lost an average of $84.43 \%$ and $81.15 \%$ water, respectively, compared with the initial moisture. According to Chiarelli et al. [24] and Sato and Cunha [22], the jabuticaba has a

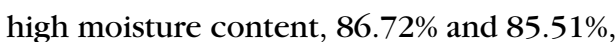
respectively; Oliveira et al. observed a variation from $69.49 \%$ to $84.66 \%$ for intact 'Sabara' jabuticaba trees from different growing regions [1].

Among the progenies evaluated, twenty percent had low water content, between $75.95 \%$ and $79.53 \%$, being a good indicator of the low perishability of the fruit of this species. Indeed susceptibility to microorganism attack increases with the increase in moisture content.

\subsection{Physicochemical and chemical characteristics}

The jabuticaba peel and pulp have a high soluble solids content, with a mean of (12.92 and 14.52$)^{\circ} \mathrm{Brix}$, respectively (table II), values close to those found by Lima et al. [(11.60 and 14.13) ${ }^{\circ}$ Brix, respectively] for the peel and pulp fractions of 'Sabara' jabuticaba tree fruit [18]. The content of soluble solids found may suggest a shorter post-harvest conservation period for jabuticaba fruit since the excess sugars in the fruit may be associated with rapid deterioration and fermentation and, consequently, reduction in shelf life [18, 23]. Besides being used as a maturation index and being decisive in maintaining flavor and aroma, soluble solids are important for in natura consumption or industrialization, as they provide better taste and higher yield in the preparation of products such as jams and jellies [6].

The total acidity of the pulp had an average of $0.85 \%$ citric acid. $100 \mathrm{~g}^{-1}$ jabuticaba tree fruits (table II). Of the progenies evaluated, twelve percent produced fruit with contents under $0.58 \%$ citric acid $100 \mathrm{~g}^{-1}$ of pulp, which confers a moderate flavor and good acceptance for in natura consumption (table II). For industrial processing, preference is given to fruits which have higher acidity, which reduces the microorganism action and avoids the addition of preservatives. The acidity values observed in jabuticaba tree pulp (Myrciaria jabuti$c a b a)$ in Diamantina corroborate the results obtained by Oliveira et al. (0.88\% to $1.65 \%$ citric acid. $100 \mathrm{~g}^{-1}$ of 'Sabara' jabuticaba tree fruit pulp) [1] and Geócze (0.92\% citric 
M.N.S. Guedes et al.

Table II.

Mean values of soluble solids, titratable acidity, [SS/TA] ratio, $\mathrm{pH}$, flavonoid and total phenols of pulp and peel fractions of jabuticaba tree fruit (Brazil).

\begin{tabular}{|c|c|c|c|c|c|c|c|c|c|c|c|}
\hline \multirow[t]{2}{*}{ Progenies } & \multicolumn{2}{|c|}{$\begin{array}{c}\text { Soluble solids } \\
\text { (SS) } \\
\left({ }^{\circ} \text { Brix) }\right.\end{array}$} & \multicolumn{2}{|c|}{$\begin{array}{c}\text { Titratable acidity } \\
\text { (TA) } \\
\text { (\% citric acid) }\end{array}$} & \multicolumn{2}{|c|}{$\begin{array}{c}{[\mathrm{SS} / \mathrm{TA}]} \\
\text { ratio }\end{array}$} & \multicolumn{2}{|c|}{$\mathrm{pH}$} & \multicolumn{2}{|c|}{$\begin{array}{c}\text { Flavonoid } \\
\left(\mathrm{mg} \text { catechin } 100 \mathrm{~g}^{-1}\right)\end{array}$} & \multirow{2}{*}{$\begin{array}{c}\text { Total phenols } \\
\left(\mathrm{g} \text { tannic acid } 100 \mathrm{~g}^{-1}\right) \\
\text { Peel }\end{array}$} \\
\hline & Pulp & Peel & Pulp & Peel & Pulp & Peel & Pulp & Peel & Pulp & Peel & \\
\hline$P_{1}$ & $16.10 \mathrm{a}$ & $13.66 \mathrm{c}$ & $0.69 \mathrm{~g}$ & $2.32 \mathrm{~b}$ & $23.22 \mathrm{c}$ & $5.96 d$ & $3.86 b$ & $3.76 \mathrm{a}$ & $0.753 \mathrm{~h}$ & $49.62 \mathrm{c}$ & 14.52 c \\
\hline $\mathrm{P}_{2}$ & $15.60 \mathrm{~b}$ & $13.03 \mathrm{~d}$ & $0.55 \mathrm{~h}$ & $1.67 \mathrm{~d}$ & $28.14 \mathrm{~b}$ & $7.81 \mathrm{c}$ & $3.84 \mathrm{~b}$ & $3.42 \mathrm{c}$ & $0.906 \mathrm{~g}$ & $32.65 \mathrm{~h}$ & $13.40 \mathrm{e}$ \\
\hline $\mathrm{P}_{3}$ & $13.90 \mathrm{~d}$ & $11.26 \mathrm{f}$ & $0.67 \mathrm{~g}$ & $1.83 \mathrm{~d}$ & $20.72 d$ & $6.15 d$ & $3.65 d$ & $3.33 c$ & $0.826 \mathrm{~g}$ & $41.93 \mathrm{e}$ & $12.64 \mathrm{f}$ \\
\hline $\mathrm{P}_{4}$ & $15.36 \mathrm{c}$ & $13.63 \mathrm{c}$ & $0.42 \mathrm{i}$ & $2.00 \mathrm{c}$ & $36.00 \mathrm{a}$ & $6.84 \mathrm{~d}$ & $3.93 \mathrm{a}$ & $3.37 \mathrm{c}$ & $1.203 \mathrm{e}$ & $41.52 \mathrm{e}$ & $13.31 \mathrm{e}$ \\
\hline$P_{5}$ & $13.60 \mathrm{e}$ & $13.00 \mathrm{~d}$ & $0.67 \mathrm{~g}$ & $0.71 \mathrm{f}$ & $20.17 d$ & $18.24 \mathrm{a}$ & $3.67 \mathrm{~d}$ & $3.26 \mathrm{~d}$ & $1.670 \mathrm{~b}$ & $32.64 \mathrm{~h}$ & $11.06 \mathrm{~h}$ \\
\hline$P_{6}$ & $14.03 \mathrm{~d}$ & $13.23 d$ & $0.77 \mathrm{f}$ & $1.77 \mathrm{~d}$ & $18.08 \mathrm{e}$ & $7.45 c$ & $3.73 \mathrm{c}$ & $2.79 \mathrm{~h}$ & $1.630 \mathrm{~b}$ & $46.13 d$ & $11.91 \mathrm{~g}$ \\
\hline $\mathrm{P}_{7}$ & $15.06 \mathrm{c}$ & $14.43 b$ & $0.58 \mathrm{~h}$ & $1.49 \mathrm{e}$ & $25.90 \mathrm{c}$ & $9.68 \mathrm{~b}$ & $3.53 \mathrm{f}$ & $3.47 \mathrm{~b}$ & $0.883 \mathrm{~g}$ & $46.57 \mathrm{~d}$ & $13.04 \mathrm{e}$ \\
\hline $\mathrm{P}_{8}$ & $14.13 \mathrm{~d}$ & $13.96 \mathrm{c}$ & $0.70 \mathrm{~g}$ & $1.52 \mathrm{e}$ & $20.07 d$ & $9.23 \mathrm{~b}$ & $3.72 \mathrm{c}$ & $3.56 \mathrm{~b}$ & $0.896 \mathrm{~g}$ & $49.76 \mathrm{c}$ & $13.36 \mathrm{e}$ \\
\hline$P_{9}$ & $14.53 \mathrm{c}$ & $13.56 \mathrm{c}$ & $0.90 \mathrm{e}$ & $1.83 \mathrm{~d}$ & $16.07 \mathrm{f}$ & $7.39 \mathrm{c}$ & $3.47 \mathrm{~g}$ & $3.34 \mathrm{c}$ & $0.726 \mathrm{~h}$ & $63.04 \mathrm{a}$ & $15.24 \mathrm{~b}$ \\
\hline$P_{10}$ & $13.43 \mathrm{e}$ & $11.43 \mathrm{f}$ & $1.07 \mathrm{c}$ & $1.75 d$ & $12.59 \mathrm{~g}$ & $6.50 \mathrm{~d}$ & $3.44 \mathrm{~h}$ & $3.35 c$ & $0.770 \mathrm{~h}$ & $28.69 \mathrm{i}$ & $10.88 \mathrm{~h}$ \\
\hline$P_{11}$ & $13.16 \mathrm{f}$ & $11.16 \mathrm{f}$ & $1.52 \mathrm{a}$ & $1.81 \mathrm{~d}$ & $8.73 \mathrm{~h}$ & $6.15 d$ & $3.33 \mathrm{j}$ & $3.21 \mathrm{e}$ & $1.250 \mathrm{e}$ & $31.64 \mathrm{~h}$ & $12.97 \mathrm{e}$ \\
\hline$P_{12}$ & $13.00 \mathrm{f}$ & $11.33 \mathrm{f}$ & $0.93 \mathrm{e}$ & $1.71 \mathrm{~d}$ & $13.95 \mathrm{~g}$ & $6.62 d$ & $3.34 \mathrm{j}$ & $3.14 \mathrm{f}$ & $1.360 \mathrm{~d}$ & $38.55 f$ & $13.97 d$ \\
\hline$P_{13}$ & $12.60 \mathrm{f}$ & $11.80 \mathrm{e}$ & $1.24 \mathrm{~b}$ & $2.77 \mathrm{a}$ & $10.16 \mathrm{~h}$ & $4.27 \mathrm{f}$ & $3.11 \mathrm{~m}$ & $3.35 \mathrm{c}$ & $0.916 \mathrm{~g}$ & $55.23 \mathrm{~b}$ & $12.53 \mathrm{f}$ \\
\hline$P_{14}$ & $14.90 \mathrm{c}$ & $12.03 \mathrm{e}$ & $0.82 \mathrm{f}$ & $2.32 \mathrm{~b}$ & $18.20 \mathrm{e}$ & $5.25 \mathrm{e}$ & $3.39 \mathrm{i}$ & $3.12 \mathrm{f}$ & $1.103 \mathrm{f}$ & $48.36 \mathrm{c}$ & $12.53 \mathrm{f}$ \\
\hline$P_{15}$ & $14.90 \mathrm{c}$ & $11.86 \mathrm{e}$ & $1.13 c$ & $2.32 \mathrm{~b}$ & $13.12 \mathrm{~g}$ & $5.10 \mathrm{e}$ & 3.231 & $3.10 \mathrm{f}$ & $1.790 \mathrm{a}$ & $42.13 \mathrm{e}$ & $13.64 d$ \\
\hline$P_{16}$ & $14.86 \mathrm{c}$ & $12.83 \mathrm{~d}$ & $0.85 f$ & $1.86 \mathrm{~d}$ & $17.53 \mathrm{e}$ & $6.88 d$ & $3.60 \mathrm{e}$ & $3.52 \mathrm{~b}$ & $0.953 \mathrm{~g}$ & $48.36 \mathrm{c}$ & $13.93 \mathrm{~d}$ \\
\hline$P_{17}$ & $14.76 \mathrm{c}$ & $13.10 \mathrm{~d}$ & $1.33 \mathrm{~b}$ & $2.17 \mathrm{c}$ & $11.02 \mathrm{~h}$ & $6.01 \mathrm{~d}$ & $3.41 \mathrm{i}$ & $3.18 \mathrm{e}$ & $1.643 \mathrm{~b}$ & $49.32 \mathrm{c}$ & $16.00 \mathrm{a}$ \\
\hline$P_{18}$ & $15.23 \mathrm{c}$ & $12.46 \mathrm{e}$ & $0.69 \mathrm{~g}$ & $2.00 \mathrm{c}$ & $22.00 \mathrm{~d}$ & $6.22 \mathrm{~d}$ & $3.49 \mathrm{~g}$ & $3.29 d$ & $0.680 \mathrm{~h}$ & $36.94 \mathrm{f}$ & $15.23 \mathrm{~b}$ \\
\hline$P_{19}$ & $16.16 \mathrm{a}$ & $14.73 b$ & $1.07 \mathrm{c}$ & $1.86 \mathrm{~d}$ & $15.10 \mathrm{f}$ & $7.88 \mathrm{c}$ & $3.53 \mathrm{f}$ & $3.51 \mathrm{~b}$ & $1.520 \mathrm{c}$ & $48.14 \mathrm{c}$ & $14.22 \mathrm{c}$ \\
\hline$P_{20}$ & $15.00 \mathrm{c}$ & $13.06 \mathrm{~d}$ & $0.81 \mathrm{f}$ & $2.05 \mathrm{c}$ & $18.39 \mathrm{e}$ & $6.35 d$ & $3.61 \mathrm{e}$ & $3.34 \mathrm{c}$ & $0.916 \mathrm{~g}$ & $38.86 \mathrm{f}$ & $12.50 \mathrm{f}$ \\
\hline$P_{21}$ & $15.00 \mathrm{c}$ & $13.36 \mathrm{~d}$ & $0.97 d$ & $1.93 \mathrm{c}$ & $15.38 \mathrm{f}$ & $6.98 \mathrm{~d}$ & $3.60 \mathrm{e}$ & $3.50 \mathrm{~b}$ & $1.226 \mathrm{e}$ & $55.08 \mathrm{~b}$ & $13.41 \mathrm{e}$ \\
\hline$P_{22}$ & $15.66 \mathrm{~b}$ & $15.60 \mathrm{a}$ & $0.73 \mathrm{f}$ & $2.33 \mathrm{~b}$ & $21.40 \mathrm{~d}$ & $6.70 \mathrm{~d}$ & $3.53 \mathrm{f}$ & $3.10 \mathrm{f}$ & $1.166 \mathrm{f}$ & $45.63 d$ & $15.61 \mathrm{a}$ \\
\hline$P_{23}$ & $15.30 \mathrm{c}$ & $14.50 \mathrm{~b}$ & $0.82 \mathrm{f}$ & $1.98 \mathrm{c}$ & $19.20 \mathrm{~d}$ & $7.30 \mathrm{c}$ & $3.73 \mathrm{c}$ & $3.39 c$ & $1.483 \mathrm{c}$ & $35.26 \mathrm{~g}$ & $10.90 \mathrm{~h}$ \\
\hline$P_{24}$ & $13.13 \mathrm{f}$ & $12.20 \mathrm{e}$ & $0.56 \mathrm{~h}$ & $2.15 \mathrm{c}$ & $23.79 c$ & $5.69 e$ & $3.39 \mathrm{i}$ & $3.31 \mathrm{~d}$ & $1.223 \mathrm{e}$ & $28.03 i$ & $13.12 \mathrm{e}$ \\
\hline $\mathrm{P}_{25}$ & $13.56 \mathrm{e}$ & $11.86 \mathrm{e}$ & $0.66 \mathrm{~g}$ & $1.41 \mathrm{e}$ & $20.44 d$ & $8.42 \mathrm{c}$ & $3.33 \mathrm{j}$ & $3.05 \mathrm{~g}$ & $1.746 a$ & $53.74 \mathrm{~b}$ & $14.12 \mathrm{c}$ \\
\hline $\begin{array}{l}\text { Coefficient } \\
\text { of variation }\end{array}$ & 2.2 & 3.03 & 6.92 & 6.24 & 8.18 & 6.65 & 0.61 & 1.14 & 4.75 & 3.10 & 1.99 \\
\hline Mean & 14.52 & 12.92 & 0.85 & 1.90 & 18.77 & 7.24 & 3.54 & 3.31 & 1.16 & 43.51 & 13.36 \\
\hline
\end{tabular}

In the columns, means followed by the same letter belong to a common group, according to the Scott-Knott criterion of grouping $(P<0.05)$.

acid $100 \mathrm{~g}^{-1}$ pulp) [25]. Machado et al. found a lower value than that observed in our study, $0.51 \%$ citric acid $100 \mathrm{~g}^{-1}$ in jabuticaba tree fruit (Myrciaria spp.) [26].

An average of $1.90 \%$ citric acid $100 \mathrm{~g}^{-1}$ peel was observed for the peel acidity, ranging from $0.71 \%$ to $2.32 \%$ citric acid $100 \mathrm{~g}^{-1}$ peel, being higher than the pulp acidity (table II). This characteristic can be very interesting regarding use of the peel for the composition of jellies and beverages, helping to raise the acidity level and thus provide greater conservation of the processed products without the addition of preservatives.

The [soluble solids/titratable acidity] (SS/ TA) ratio is one of the most used forms for 
assessing the fruit flavor, being more representative than isolated measurement of sugars or acidity [6]. We found that $20 \%$ of the progenies obtained the best pulp SS/ TA ratio scores (table II), which provides the fruit with its great flavor. The average observed for the jabuticaba tree accessions' pulp SS/TA ratio was 18.77 ; it is higher than the results found by Geócze [25] and Soprano and Koller [21], who found average SS/TA values of 16.30 and 11.40 , respectively.

The mean SS/TA ratio for the peel was 7.24 (table II). The values found in the tropical region of altitude are similar to those found by Lima et al. for the peel of jabuticaba tree fruit (Myrciaria jabuticaba) cultivated in the Lavras-MG region with similar climatic characteristics [18].

The average $\mathrm{pH}$ values were 3.54 for the pulp and 3.31 for the peel (table II). Similar values were found by Sato and Cunha [22], Geócze [25] and Oliveira et al. [1] 3.22, 3.50 and 3.42, respectively, for whole jabuticaba fruit. Lima et al. found $\mathrm{pH}$ values of 3.50 and 3.39, respectively, for the pulp and peel of 'Sabara' jabuticaba tree fruit fractions [18], values similar to those found in our work.

The average flavonoid levels were higher in the peel fraction $(43.51 \mathrm{mg}$ catechin. $100 \mathrm{~g}^{-1}$ ) than the pulp fraction (1.16 $\mathrm{mg}$ catechin $100 \mathrm{~g}^{-1}$ ) (table II), results similar to those found by Pinto et al. [(87.80 and 2.96) $\mathrm{mg}$ catechin $\cdot 100 \mathrm{~g}^{-1}$, respectively, in the peel and pulp fractions of jabuticaba tree fruit] [27]. Rufino et al. found concentrations of $(147 \pm 42.5) \mathrm{mg}$ yellow flavonoids $100 \mathrm{~g}^{-1}$ in fresh jabuticaba tree fruits (Myrciaria cauliflora) [28]. Flavonoids are polyphenolic structures of lower molecular weight that are found naturally in plants; they are responsible for the color aspect of leaves, flowers and fruits [29].

The levels of flavonols are more concentrated in the peel than in the pulp, suggesting the use of the jabuticaba peel as a potential source of flavonoids. According to Tsuchiya, the antioxidant capacity of flavonoids confers therapeutic potential for the treatment of cardiovascular diseases including ulcers and gastrointestinal tract cancer [30].

The mean values of total phenols ranged from (10.88 to 16.00$) \mathrm{g}$ of tannic acid $100 \mathrm{~g}^{-1}$ of fresh peel. Lima et al. found $11.99 \mathrm{~g}$ of tannic acid. $100 \mathrm{~g}^{-1}$ of fresh peel in 'Sabara' jabuticaba fruit [18], similar to the values found in our study and higher than those found in most fruit pulp consumed in Brazil, such as acerola, mango, blackberry and grape, according to Kuskoski et al. [31]. Acai would have (1.82 to 5.98) $\mathrm{g}$ tannic acid $100 \mathrm{~g}^{-1}$ of fresh fruit [8].

Rufino et al. found $(440 \pm 9.9) \mathrm{mg}$ gallic acid Eq. $100 \mathrm{~g}^{-1}$ for jabuticaba fruit fresh matter [28]. The peel of the jabuticaba tree fruit is characterized by having a strong astringency determined by the presence of soluble phenolic compounds, mainly tannins.

The pulp fraction of jabuticaba fruit evaluated in our study showed low amounts of flavonoids, and an absence of tannic acid, by the methodology used. This result corroborates with those obtained by Roesler et al., who detected the best total phenolic contents for skin and seed fractions and lower concentrations for the pulp fraction of different cerrado fruits [32].

According to Malacrida et al. [33] and Rufino et al. [28], the total phenolic content varying considerably from one fruit to another depends on the part of the fruit examined and the extraction method. Polyphenolic compounds found in plants, foods and beverages are used as a source of raw material for various pharmaceutical and food industries.

Tannic acid belongs to the group of polyphenols that are known to inhibit the growth of selected tumors [34]. According to Lima et al., tannins can interact with dietary proteins and form insoluble complexes, which may cause damage to health, such as reduced protein digestibility, enzyme inhibition, growth restriction, and others, but can have various beneficial effects [18]. Therefore, the characterization of such constituents is of utmost importance in order to provide safety in the use of the whole jabuticaba fruit, as well as its fractions, in the food industry. 
The nutraceutical market value of jabuticaba tree fruits lies in their high content of phenolic compounds. These are connected to intracellular antioxidant mechanisms and antioxidant acting enzymes, contributing to the prevention of chronic diseases resulting from oxidative stress [35]. Flavonoids, phenolic acids and tannins play important ecological roles primarily in attracting pollinators, and act as defense compounds against microbial infections [36].

\section{Conclusion}

There is variability in the characteristics analyzed, allowing the economic exploitation of the jabuticaba tree fruit for in natura consumption and processing.

The variables fruit mass, [soluble solids/ titratable acidity] ratio of the pulp fraction, $\mathrm{pH}$ of the peel fraction, peel and pulp flavonoid fractions, and total phenols can be considered the best characteristics to represent the genetic diversity among the jabuticaba tree progenies, since they formed the highest number of groups.

Five progenies $\left(\mathrm{P}_{1}, \mathrm{P}_{2}, \mathrm{P}_{4}, \mathrm{P}_{22}\right.$ and $\left.\mathrm{P}_{23}\right)$ among those studied were superior to the others regarding fruit yield, soluble solids and SS/TA, presenting low acidity: all agronomic characteristics of interest to the in natura market.

Five other progenies $\left(\mathrm{P}_{8}, \mathrm{P}_{9}, \mathrm{P}_{13}, \mathrm{P}_{16}\right.$ and $P_{17}$ ) presented fruits with high flavonoid content in the peel and increased peel yield, agronomic characteristics favorable for industrial use.

\section{References}

[1] Oliveira A.L., Brunini M.A., Salandini C.A.R., Caracterização tecnológica de jabuticabas 'Sabará' provenientes de diferentes regiões de cultivo, Rev. Bras. Frutic. 25 (2003) 397400.

[2] Pereira M.C.T., Salomão L.C.C., Mota W.F., Vieira G., Atributos físicos e químicos de frutos de oito clones de jabuticabeiras, Rev. Bras. Frutic. 22. (2000) 16-21.
[3] Reynertson K.A., Yang H., Jiang B., Basile M.J., Kennelly E.J., Quantitative analysis of antiradical phenolic constituents from fourteen edible Myrtaceae fruits, Food Chem. 109 (2008) 883-890.

[4] Legatti A.V.L., Batista A.G., Dragano N.R.V., Marques A.C., Malta L.G., Riccio M.F., Eberlin M.N., Machado A.R.T., Silva L.B de C., Ruiz A.L.T.G., Carvalho J.E. de., Pastore G.M., Júnior M.R.M., Jaboticaba peel: Antioxidant compounds, antiproliferative and antimutagenic activities, Food Res. Int. 49 (2012) 596-603.

[5] Fagundes R.G.,Yamanishi K.O., Características físicas e químicas de frutos de mamoeiro do grupo 'Solo' comercializados em 4 estabelecimentos de Brasília-DF, Rev. Bras. Frutic. 23 (2001) 541-545.

[6] Chitarra M.I.F., Chitarra A.B., Pós-colheita de frutos e hortaliças: fisiologia e manuseio, UFLA, Lavras, Brazil, 2005.

[7] Haponik C.A., Rebolças A.F., Paiva W.O., Almeida A.S. de., Mosca J.L., Silva de O., Alves R.E., Seleção de Progênie de Melões 'Tupã' para a Qualidade e Valor Nutricional, Proc. Interam. Soc. Trop. Hortic. 47 (2003) 58-60.

[8] Santos G.M., Maia P.M.S., Costa J.M.C., Figueiredo R.W., Prado G.M, Correlação entre a atividade antioxidante e compostos bioativos de polpas de açaí comerciais de açaí (Euterpe oleracea Mart.), Organo Of. Soc. Latinoam. Nutr. 58 (2008) 187-192.

[9] Citadin I., Vicari I.J., Silva T.T., Danner M.A., Qualidade de frutos de Jabuticabeira (Myrciaria cauliflora) sob influência de duas condições de cultivo: sombreamento natural e pleno sol, Rev. Bras. Agrociênc. 11 (2005) 373-375.

[10] Aikpokpodion P.O., Assessment of genetic diversity in horticultural and morphological traits among papaya (Carica papaya) accessions in Nigeria, Fruits 67 (2012) 173-187.

[11] Jakopic J., Slatnar A., Stampar F., Veberic R., Simoncic A., Analysis of selected primary metabolites and phenolic profile of 'Golden Delicious' apples from four production systems, Fruits 67 (2012) 377-386.

[12] Cupolillo F., Diagnóstico hidroclimatológico da Bacia do Rio Doce, Univ. Fed. Minas Gerais, Tese, Belo Horizonte, Brazil, 2008, $156 \mathrm{p}$.

[13] Anon., Official methods of analysis, 12th ed., Assoc. Off. Anal. Chem. (AOAC), Wash., U.S.A. 
[14] Anon., Normas analíticas do Instituto Adolfo Lutz: Métodos químicos e físicos para análise de alimentos, 5th ed., Inst. Adolfo Lutz, São Paulo, Brazil, 2008.

[15] Zhuang X.P., Lu Y.Y., Yang G.S., Extraction and determination of flavonoid in ginkgo, Chin. Herb. Med. 23 (1992) 122-124.

[16] Goldstein J.L., Swain T., Changes in tannins in ripening fruits, Phytochemistry 2 (1963) 371-383.

[17] Ferreira D.F., Sisvar: a computer statistical analysis system, Ciênc. Agrotecnol. 35 (2011) 1039-1042.

[18] Lima A de. J.B., Corrêa A.D., Alves A.P.C., Abreu C.M.P., Barro A.M.D., Caracterização química do fruto jabuticaba (Myrciaria cauliflora Berg) e de suas frações, Soc. Latinoam. Nutr. 58 (2008).

[19] Jesus N de., Martins A.B.G., Almeida E.J. de, Caracterização de quatro grupos de jabuticabeira, nas condições de Jabuticabal-SP, Rev. Bras. Frutic. 26 (2004) 482-485.

[20] Hickel E.R., Espessura da polpa como condicionante do parasitismo de mosca-dasfrutas (diptera:tephritidae) por hymenoptera:braconidae, Ciênc. Rural 32 (2002) 1005-1009.

[21] Soprano E., Koller O.L., Avaliação de frutos de espécies frutíferas tropicais com potencial para cultivo em Santa Catarina, XX Congr. Bras. Frutic. 2008, Anais, Vitória/ES, Brazil, 2008.

[22] Sato A.C.K., Cunha R.L., Influência da temperatura no comportamento reológico da polpa de jabuticaba, Ciênc. Tecnol. Aliment. 27 (2007) 890-896.

[23] Alves A.P de C., Corrêa A.D., Pinheiro A.C.M., Oliveira F.C., Flour and anthocyanin extracts of jaboticaba skins used as a natural dye in yogurt, Int. J. Food Sci. Technol. 48 (2013) 2007-2013.

[24] Chiarelli R.H.C., Nogueira A.M.P., Venturin F.W.G., Fermentados de jabuticaba (Cauliflora Berg.): Processos de produção, características físico-químicas e rendimento, Braz. J. Food Technol. 8 (2005) 277-282.

[25] Geócze A.C., Influência da preparação do licor de jabuticaba (Myrciaria jabuticaba Vell Berg) no teor de compostos fenólicos, Fac. Farm. UFMG, Tese, Bras., 2007, 81 p.
[26] Machado N.P., Coutinho E.F., Caetano E.R., Embalagens plásticas e refrigeração na conservação pós-colheita de jabuticaba, Rev. Bras. Frutic. 29 (2007) 166-168.

[27] Pinto N.A.V.D., Moreira W.A., Cardoso L. de M., Pantoja L.A., Jaboticaba peel for jelly preparation: an alternative technology, Ciênc. Tecnol. 31 (2011) 864-869.

[28] Rufino M. do S.M., Alaves R.E., Brito E.S. de, Jiménez J.P., Calixto F.S., Mancini F.J., Bioactive compounds and antioxidant capacities of 18 non-traditional tropical fruits from Brazil, Food Chem. 121 (2010) 996-1002.

[29] Volp A.C.P., Renhe I.R.T., Barra K.B., Stringueta P.C., Flavonóides antocianinas: características e propriedades na nutrição e saúde, Rev. Brás. Nutr. Clin. 23 (2008) 141149.

[30] Tsuchiya H., Structure-dependent membrane interaction of flavonoids associated with their bioactivity, Food Chem. 120 (2010) 1089-1096.

[31] Kuskoski E.M., Asuero A.G., Troncoso A.M., Mancini F.J., Fett R., Aplicación de diversos métodos químicos para determinar actividad antioxidante en pulpa de frutos, Ciênc. Tecnol. Aliment. 25 (2005) 726-732.

[32] Roesler R., Malta L.G., Carrasco L.C., Holanda B., Sousa C.A.S., Pastore G.M., Atividade antioxidante de frutas do cerrado, Ciênc. Tecnol. Aliment. 27 (2007) 53-60.

[33] Malacrida C.R., Ângelo P.M., Jorge D.A., Composição química e potencial antioxidante de extratos de sementes de melão amarelo em óleo de soja, Rev. Ciênc. Agron. 38 (2007) 372-376.

[34] Weston R.J., Bioactive products from fruit of the feijoa (Feijoa sellowiana, Myrtaceae): A review, Food Chem. 121 (2010) 923-926.

[35] Franco M.C.C., Chimal C.R., Hernández M.G.H., Colín C.A.N., Martínez M.A.H., Maldonado S.H.G., Physicochemical, nutritional and health-related component characterization of the underutilized Mexican serviceberry fruit [Malacomeles denticulata (Kunth) G.N. Jones], Fruits 69 (2014) 47-60.

[36] Middleton Jr. E., Kandaswami C., Theoharides T.C., The effects of plant flavonoids on mammalian cells: Implications for inflammation heart disease and cancer, Pharm. Rev. 52 (2000) 673-751. 
M.N.S. Guedes et al.

\section{Calidad del fruto de descendencias de jabuticabas cultivados en un clima tropical de altitud.}

Resumen - Introducción. El jabuticaba (Myrciaria jabuticaba) pertenece a la familia de las Mirtáceas; produce pequeños frutos y es autóctono de las regiones del centro, sur y sureste de Brasil. Material y métodos. Se evaluaron las características físicas, físico-químicas y químicas de frutos de 25 descendencias jabuticaba, desarrolladas en un clima tropical montañoso: peso, diámetros longitudinal y transversal del fruto, rendimientos de piel y pulpa, masa de las semillas, número de semillas por fruto, índice de humedad de la pulpa y de la piel, contenido de sólidos solubles, acidez valorable, índice [sólidos solubles/acidez valorable], $\mathrm{pH}$, contenidos de flavonoides y compuestos fenólicos. El dispositivo experimental fue totalmente aleatorio, se estudiaron los tratamientos compuestos por las 25 descendencias de jabuticaba. Resultados. Se observó un efecto significativo de todas las características evaluadas entre las descendencias. La composición química reveló que la piel del fruto del jabuticaba tiene un contenido elevado de compuestos fenólicos y un índice importante de flavonoides. Nuestros resultados indican que existe variabilidad entre las características analizadas, lo que justifica una explotación económica de los frutos del jabuticaba para su consumo fresco y para su industrialización. Conclusión. El estudio combinado de las características físicas y químicas se puede proponer como una excelente herramienta para seleccionar descendencias superiores. Los frutos de cinco descendencias revelaron características interesantes para el mercado de frutas frescas, mientras que los frutos de otras cinco descendencias que presentan altos contenidos de antioxidantes naturales podrían ser una buena opción para la industria alimentaria.

Brasil / Myrciaria jabuticaba / frutas / propiedades fisicoquímicas / flavonoides / contenido fenólico / procesamiento 